\title{
Increasing the Advertising Literacy of Primary School Children in Ireland: Findings from a Pilot RCT
}

\author{
V. O’Rourke, S.J. Miller, L. Dunne \\ Queen's University Belfast, $U K$
}

\begin{abstract}
This study reports the effect of four lessons of a recently developed Irish media literacy teaching intervention on the advertising literacy of children aged 8-11. Covariates of age and gender were also considered. Alongside this, a process evaluation was completed. The results of this pilot RCT show that children aged between 8 and 11 are capable of increasing their knowledge regarding the persuasive intent and the selling intent of marketing messages. The intervention had a statistically significant positive impact on advertising literacy. This study finds no evidence to suggest that advertising literacy is gendered. Qualitative discussions indicate that the teaching materials were well received by both teachers and children. The need for regular advertising literacy lessons for children was unearthed. The challenge of attempting to include more content in an increasingly crowded curriculum was cited as the main barrier to delivering regular media literacy lessons. Recommendations for increased media literacy education in primary school are discussed. These findings are of interest to parties including educators, parents, policy makers and marketers.
\end{abstract}

\section{Introduction}

Digital communication technologies create multiple platforms for learning, entertaining as well as educating children, yet they also change the nature of commercial communications with children. Consumption of digital communication technologies is individualistic in manner. Children's consumption of media has doubled over the past ten years. Although challenging to measure due to the multiplicity of media and its simultaneity of consumption, it is reported that, at a minimum, $74 \%$ of children aged between 8 and 11 are spending approximately 36 hours per week online, gaming and watching television [1]. Commercial organisations can communicate directly with children in the absence of parental guidance [2]. The majority of digital content, including peer communications and games, are in some way commercially funded [3]. Content creators have moved from traditional, regulated, broadcasting channels to content curators such as YouTube. Rather than maturing in an independent fashion from the commercial world, organisations can bypass parents and directly communicate with children, developing the consumer from an ever-decreasing age. Media communicate unrealistic ideals of life [4]; messages promoting the essentiality of acquisition and the extension of self through ownership pervade media. The ownership of material goods is promoted as a mechanism by which consumers can achieve success, personal happiness and self-fulfillment. Fueled by this, the consumption of branded goods defines Western society [5]. From a very young age children are aware of brands.

\section{Regulating and educating for the digital media landscape}

Historically, commercial messages were one-way via the medium of a shared television which parents could 'police' more effectively, whereas children are now exposed to a multitude of content. Content creators may not always be cognisant of their young consumers' developing cognitive defences. Children may be at risk of being manipulated in a manner which is inappropriate. Regulation of digital advertising in its many guises is proving ever more problematic [6;7]. Regulation has evolved from protectionism to a libertarian view, which has resulted in freedom for commercial enterprises to communicate directly with children. Regulating the media industry is challenging and self-regulation efforts have proven ineffective. Legislative regulation, if tested, would inevitably move the focus of arguments to points of law rather than the interest of children [8]. Co-regulation may be a positive approach, education offers another.

Media literacy policy developments within the European Union take a two-pronged approach [9]. The role of regulatory bodies is promoted through codes of conduct, developing awareness, and promoting research with a view to supporting the inclusion of media literacy as core curriculum. Alongside this, actors within the media industry are requested to develop awareness campaigns to inform citizens of the media process and its economic consequences. In response, a new European initiative, The Media Literacy week, was held from 18-22 March 2019 [10]. During this time in Ireland the 'Be Media Smart' campaign was launched by Media Literacy Ireland (MLI), to promote informed consumption of media messages. 
Traditionally perceived as being free from market forces, schools themselves have become commercialised through funded projects, classroom materials, lunch items, and other brands introduced in the playground $[8 ; 11 ; 12]$. Children are part of consumer culture. If schools do not integrate consumer culture studies of some form into curriculum, there is a danger that children will perceive the educational system to operate separate to the consumer world they inhabit. This will enable a narrative whereby commercial enterprises 'know best' and the education system does not favour the contemporary interests of children [12]. Prohibition of marketing messages is futile in modern society [13]. Media literacy education is essential so that young consumers are enabled to critically evaluate advertising messages and develop the skills required to navigate contemporary consumer culture $[14 ; 15]$.

Children are agentic and capable of informed decision making [16]. A growing number of researchers $[11 ; 12 ; 17 ; 18]$ contend that education systems must reconsider curriculum to accommodate contemporary consumer culture. The gap between protectionism and empowerment is bridged through media literacy education. The aim of media literacy education is to inform individuals so that they can become critical consumers of media. Specifically, advertising literacy skills provide counterbalance to marketing appeals. Developing cognitive defences in children enables informed assessment of marketing messages $[17 ; 19]$.

\section{Advertising literacy}

Drawing on the work of Friestad and Wright [20] and Petty and Cacioppo [21] amongst others, Rozendaal et al. [22] take a broad view of advertising literacy. They propose that advertising literacy is a multi-dimensional construct, comprising conceptual advertising literacy and attitudinal advertising literacy. As children are cognitively developing and because they process messages affectively, low elaboration occurs. Consequently, critical evaluation of messages is absent. Thus behaviour may not follow in a logical manner. There is developing debate that this affective element of advertising literacy is that which determines behavioural intention $[22 ; 23 ; 24 ; 25]$. Research in this regard is emerging [17]. In line with the Message Interpretation Process (MIP) model [26], Rozendaal et al.'s [22] representation of advertising literacy acknowledges that children can gain enjoyment and entertainment from advertising messages as well as potentially experiencing adverse effects.

No single comprehensive conceptualisation of advertising competences exists [27]. Any definition of children's media literacy capabilities should acknowledge if the child can process advertising messages in a critical manner [28]. Aligned to Piaget's developmental stages, Livingstone and Helsper [29] surmise three stages of advertising literacy in children. Before the age of 5 children are unable to distinguish marketing messages from content, and regard them as entertainment. From the age of 7 children begin to recognise the persuasive intent of marketing messages, although they may not apply this knowledge in a fruitful manner. Enjoyment of advertisements depreciates the motivation to activate message resistance strategies [6]. Until the age of 11 children are capable of developing conceptual advertising literacy, attitudinal elements develop from this age onward [30]. From the age of 12, children have the developed a critical understanding of the motivations of commercial messages and may be skeptical or distrustful of such marketing messages.

Positioned within the concept of persuasion knowledge, advertising literacy influences the persuasion process in children [29; 31]. Advertising literacy is the knowledge and abilities an individual possesses to process various advertising tactics [24]. It is a 'form of literacy of media messages that typically concentrates on persuasion but also offers information and/or entertainment' [32, p169]. Pertinent for this study, Hudders et al. [24, p911] recapitulate definitions of cognitive advertising literacy as:

(a) 'the ability to distinguish advertising content from regular media content', and (b) 'the ability to understand the underlying commercial intent and knowledge of the techniques used by advertising ... [this can be] subdivided into two separate sub skills: individuals' understanding of the selling intent of advertising and ... of the persuasive intent of advertising'.

It should not be taken for granted that children have the ability to critically assess advertising messages. Endeavours to promote advertising literacy amongst children must consider the extent to which children can differentiate between message senders, the persuasion tactics being used by advertisers, and advertising effects [22]. Notable for this study, of studies that examine cognitive, attitudinal and behavioural responses to advertisers intended effects, cognitive responses are the least researched [33].

\section{Media literacy education}

The MIP model [26] offers a contemporary cognitive approach to media literacy education and addresses the issues inherent in teaching advertising literacy. The MIP model teases out the relationship between attitudes towards media messages and behavioural intention, in so far as it accommodates 
for situations where affective evaluations may override cognitions - a common occurrence amongst children [28]. The notion of social norm is extended to include the degree to which that norm reflects an individual's personal experiences and customs [34]. The truthfulness of the message is assessed. Logical comparisons are made between descriptive norms and what is observed in the media. If media messages are perceived as desirable, consequences of performing the behaviour are then considered before a behaviour is exhibited. Identification with references groups in the message (cartoon characters, celebrities and sporting heroes, or user generated content) results in an expectation that conforming to the behaviours suggested in the message will bring positive consequences. Over time the elaboration required diminishes. Heuristics are employed to accept or reject the message senders' appeals. Logical heuristics include message sender credibility, perceived realism and the appeal's congruence with a pertinent reference group. Affective heuristics include the desirability of the message [26]. This model makes an important contribution to the teaching of media literacy [28; 34]. It draws attention to the fact that that decision making is not a straightforward process. On occasion emotion can dominate decision making, thus logic is suspended [26]. Promoting and reinforcing logical heuristics is a requirement of media literacy interventions.

\subsection{Media literacy interventions}

The number of media literacy interventions delivered across various contexts has grown $[32 ; 35]$. For the most part they have been found to be effective in addressing adverse impacts of media messages. In their meta-analysis of the effects of media literacy interventions, Jeong et al. [36] found that effects are greater on media related outcomes such as knowledge $(\mathrm{d}=1.12, \mathrm{p}<.001,95 \% \mathrm{CI}: 0.77$ to 1.47) and attitudes (d=.28, p<.001, 95\% CI: 0.17 to 0.39 ) as opposed to behaviour related outcomes $(\mathrm{d}=.23, \mathrm{p}<.001,95 \% \mathrm{CI}: 0.15$ to 0.31$)$. These findings may be due to the focus of interventions on critical thinking, or the fact that behaviour related outcomes are more latent in nature.

Rozendaal et al.'s [31] empirical work found evidence to support Friestad and Wright's [20] 'change of meaning' concept. They advocate for educational interventions that focus on developing persuasion knowledge. 'To warrant a fair commercial environment for children, it is of great importance for policy makers to base their policies concerning children and advertising on scientific insights in children's development of advertising literacy' [31; p346]. Advertising literacy interventions highlighting the persuasive intent of organisations are indispensable. Rather than inoculation against negative effects, in line with the objective of Friestad and Wright's Persuasion Knowledge Model (PKM), advertising interventions should seek to increase persuasion knowledge. Advertising literacy will empower children to critically evaluate commercial messages and make informed choices [28; 34]. As per Austin's [26] MIP model, interventions targeting logic and emotional aspects of information processing will engender in children a propensity to be skeptical of marketing messages.

Media literacy literature advocates media literacy interventions to develop critical thinking amongst children, and to reduce the triggering of effects. Potter [37] surmises that literature in this regard is developing at present and, interestingly, as of yet, few studies have addressed the teaching of advertising literacy in schools $[32 ; 37]$. Walther et al. [38] contend that if children are educated and enabled to process media messages in a healthier way, this may result in a reduction in media exposure, increased critical thinking, and positive behaviour change.

\subsection{Media literacy in Irish curriculum}

Revisions of materials for primary curriculum in Ireland reflect the changing agenda in terms of childhood education [39]. The promotion of wellbeing forms an essential component of the modern Irish curriculum. In the Social, Personal and Health Education (SPHE) module, wellbeing is separated into three strands; myself, myself and others, and myself and the wider world. Educators are encouraged to include aspects of the three strands into their teaching. Within the 'myself and the wider world' strand, media education is one unit. The extent to which any strand is addressed in a primary school is at the behest of individual school policy. There are a number of SPHE teaching resources available for educators [40; 41]. Resources focus on topics including social and emotional skills and citizenship, awareness of substance abuse, and internet safety. Currently, by means of the 'Stay Safe Programme', the substantive focus of media literacy education is on safe practices when using the internet and social media. This personal safety skills programme is delivered in all primary schools in Ireland. This is the minimum amount of media literacy education a primary school pupil is currently exposed to. While digital media literacy is essential, multiple media literacies (entailing critical awareness of production, representation, language, and audience) are vital. 'Education about the media should be seen as an indispensable prerequisite for education with or through the media' [42].

Although it may prove challenging to incorporate multiple aspects of media literacy into a crowded curriculum [43], teaching advertising literacy as an 
essential component of contemporary primary curriculum is advocated $[29 ; 31 ; 44 ; 45 ; 46]$. This will help to ensure that the gap between children's educational worlds inside and outside of the classroom are more closely aligned [47]. Reflecting emerging concerns regarding the impacts of consumer culture on children, specifically of marketing appeals, an Irish media literacy teaching intervention, MediaWise, was developed by Safefood in 2017.

\section{Methodology}

The findings presented herein are an element of a wider study appraising the effect of a media literacy intervention in primary curriculum on outcomes of advertising literacy, materialism and subjective wellbeing. A mixed methods approach with a predominantly quantitative focus was employed. The research was conducted in two phases. Phase one was quantitative, and comprised a pilot randomised controlled trial (RCT) $(n=324)$ carried out between the months of January and May 2018. Phase two, a process evaluation, employed qualitative methods to explore perceptions of delivering media literacy lessons in the classroom. It consisted of interviews with six teachers and two focus groups with 17 children, carried out during the months of April and May 2018.

\subsection{Pilot RCT}

This study was a small scale educational evaluation and the design characteristics [37] were as follows: the teacher was the agent who delivered the intervention, children aged between eight and 11 in third and fourth class in primary schools in the Republic of Ireland were the target, the programme consisted of four lessons of the third and fourth class MediaWise media literacy teaching intervention. This paper reports findings relating to the advertising literacy outcome.

Gender and age were identified as covariates [48; 49]. Females hold stronger stereotypes, are more susceptible to emotional influences and have stronger social motivations for consumption. Males are more likely to be influenced by non-personal communications and more influenced by functional appeals [50]. Reported earlier, as children mature their cognitive abilities to assess marketing messages become more sophisticated. From the age of 7 onward children are able to consider consumption in symbolic terms and their behaviour begins to solidify by the age of 12 [51;54]. Similarly, from the ages of 8 to 10 there is a substantial development in children's persuasion knowledge [31]. Children aged between 8 and 11 are typically in third class (year 5) and fourth class (year 6) in primary school in Ireland.
The MediaWise resource aligns well to this age group as it provides materials explicitly designed for these two classes combined. The following hypotheses were developed: H1: a media literacy intervention can increase advertising literacy when pre-test advertising literacy scores, gender and age are controlled for. Alongside the main regression model, in order to explore if there was an interaction effect between pre-test advertising literacy scores and the intervention, hypothesis 2 was developed: H2: there is an interaction between the intervention and pre-test advertising literacy scores that helps to predict post-test advertising literacy scores.

A growing number of materials for media literacy educational interventions are available $[53 ; 54 ; 55]$. Informed by these established materials, a free online educational resource, MediaWise, was developed for the Irish primary school curriculum. Its aim is to 'enable children to appraise, interpret and analyse messages, enabling children to take informed choices' [56]. Aligned to the Broadcasting Authority of Ireland's [57] core competency framework, the intervention maps to those learning outcomes related to advertising literacy in the media education unit of the SPHE curriculum. The resource is also linked to curricula including maths, visual arts and language. The materials facilitate a generic approach and provide multiple contexts for learning, they are spiral in nature and learning is activity-based [58]. The resource encompasses recommendations from a previous evaluation study of primary school media literacy materials [11] Worksheets are plentiful, clear instruction for teachers is provided, and current advertising examples are included in the resource. The design of MediaWise follows Potter's [59] guidelines; it is informed by extant literature, has been pilot tested, includes relevant materials, and does not appear cluttered. Piloted with teachers before launch, its effectiveness in a classroom setting had yet to be explored. Previous studies $[32 ; 36]$ and practicalities arising from time constraints in a crowded curriculum informed the decision to employ four lessons in this study.

The contents of the four lessons employed were as follows; Lesson one seeks to enable children to recognise the omnipresence of media and to understand the motivations of advertisers. Lesson two's objective is to understand that everyone has a point of view. Lesson three enables children to recognise different elements that are used in the media and to explain how they can affect emotions. The objective of Lesson 4 is to differentiate between a need and a want.

The study was conducted during the months of January and May 2018. Pre-test data collection took place between the 16-01-2018 and 7-02-2018. It was anticipated that post-test data collection would take place between six and eight weeks later. Post test data was collected between 13-03-2018 and 2-05- 
2018. Unavoidable delays in post-test data collection occurred as a consequence of unplanned school closures due to adverse weather, scheduled events, and term holidays in the school calendar.

A pen and paper based personal survey measured baseline outcomes. Prior to data collection, the questionnaire was piloted to assess the ease of interpretation of questions and to determine the average completion time (20 minutes). The researcher remained present while children completed the questionnaire to safeguard against any unintended coercion to provide 'correct' responses [60].

The study was conducted across seven primary schools in the Republic of Ireland, selection of schools was purposive, in order to include a geographic and socioeconomic spread of respondents. To increase similarity between the groups, stratified randomisation was carried out at a school level by means of paired allocation on the basis of school size. A repeated measures final sample size of 324 was achieved: 200 respondents were in the intervention group and 124 respondents were in the control group.

The Consolidated Standards of Reporting Trials (CONSORT) statement guides the following presentation of the characteristics of the intervention [61]. The intervention providers were the teachers who voluntarily agreed to take part in the study. The objectives of the intervention were communicated to teachers during the process of obtaining consent from all research parties. Materials were not discussed verbally with teachers until after baseline data was collected. At this time, schools were informed of their group allocation, no masking took place. Each teacher in the intervention group received verbal instruction along with an individual lesson pack. Contained within the lesson pack was a coversheet outlining the purpose of the study, a copy of the four lesson plans, copies of the associated worksheets and four intervention record proforma. Lessons were delivered during the weeks from 01 02-2018 to 26-04-2018. The intervention was delivered with relatively high fidelity. While there was attrition in the number of lessons delivered, seven of the nine teachers delivered 75 percent of the lessons and just under half of the teachers delivered all four lessons. The intervention teaching materials can be accessed here: https://www.safefood.eu/Education/Primary(ROI)/MediaWise.aspx. Teachers in the control group received a copy of the intervention materials after post-test data collection was completed. The design of the study did not facilitate concealing group allocation, during the trial no placebo was administered to the control group. No changes were made after the trial commenced. By means of multiple regression modelling, the impact of a media literacy intervention on advertising literacy was considered.

Measures of media literacy are developing [62]. The number of dispositional scales designed for children are limited. Similar to others [32], the scale employed was adapted from the work of Rozendaal et al. [22]. Those elements of their Conceptual Advertising Literacy Scale (CALSc) which did not require video as part of the data collection process, therefore measuring dispositional conceptual advertising literacy, were utilised. The initial Cronbach's alpha for the adapted scale indicated the need for refinement of the measure. Reducing the scale to six items which measured two subcomponents; 'understanding selling intent' and 'understanding persuasive intent', resulted in a Cronbach's $\alpha$ of .53 pre-test and .66 post-test. These two components measure one of the two key abilities of cognitive advertising literacy as defined by Hudders et al. [24] - the ability to understand the underlying commercial intent of advertising.

This measure of conceptual advertising literacy aligns with the learning outcomes of the MediaWise intervention; "children should be able to ... recognise the different media they encounter ...; recognise most advertising; ...; identify that advertisers pay to communicate their message [and] know the four aims of advertising; tell us something we need to know, persuade us to buy a product, persuade us to change our behaviour and put forward a point of view' [57]. Furthermore, this measure aligns well to the learning outcomes for the media education unit of the 'myself and the wider world' strand of SPHE in Irish primary curriculum [58]. Given that the measure is somewhat constrained as it was not possible to employ the entire scale and measure situational elements of persuasion knowledge, a moderate reliability of over .5 was considered acceptable [63], and sufficient to facilitate analysis. Furthermore, the Inter Class Correlation (ICC) coefficient (ICC $=0.44,95 \%$ CI $[.31, .55]$ ) indicated that the test-retest reliability of the advertising literacy measure was fair [64].

Data were analysed using SPSS v.24. Outcome measures were standardised preceding analysis. Multiple regression modelling enabled the assessment of the impact of the intervention [65] when controlling for pre-test advertising literacy scores. Covariates of gender and age were measured on nominal scales and their impact on the relationship modelled was explored by means of dummy variables. The 'class' variable acted as the covariate of age as, in line with the typical demographics of class compositions, the respondent profile indicates that younger children in the sample were in third class while older children were in fourth class. 


\subsection{Process evaluation}

Qualitative discussions with children and teachers took place on school grounds directly after post-test data collection was completed. Discussions lasted on average 30 minutes. Judgement sampling was utilised to select schools to conduct focus groups with children in. Two schools that had delivered all four lessons were invited to participate. Eight children from one school and nine children from a second school took part in group discussions. All teachers who delivered the intervention were invited to take part in a depth interview. Six teachers who delivered the intervention shared their experiences in delivering the intervention. Three teachers were employed in the same school and elected to take part in a group interview. The other three teachers, from separate schools, were interviewed individually. Qualitative data were transcribed and analysed using thematic analysis. In line with the qualitative research objectives, data were sorted, reviewed and classified into emerging themes [66]. This process evaluation provided a rich understanding of the context for the effects uncovered in the trial.

The project received the approval of the Research Ethics Committee of Queen's University, Belfast in November 2017. All parties - schools, teachers, parents and children - were informed and consented to take part in the research. Active consent to take part in the research was gained from the school, from the parent/ guardian, from the child and from teachers.

\section{Findings}

\subsection{Sample characteristics}

$54.3 \%$ of the sample were girls $(n=148)$ while $45.7 \%$ were boys $(n=176)$. Just over half of the sample $(51.5 \%)$ were in fourth class $(n=167)$ while $48.5 \%$ were in third class $(n=157)$. The age range of respondents was 8-11 years. The mean age of children in third class was 8.8 years $(\mathrm{SD}=.44)$ and the mean age of children in fourth class was 9.7 years $(\mathrm{SD}=.51)$.

\subsection{Results}

Table 1. Descriptive statistics for advertising literacy

\begin{tabular}{|l|l|l|l|l|}
\multicolumn{7}{|c}{ pre-test and post-test } \\
\hline Outcome & Min \& Max & $\begin{array}{l}\text { Total Mean } \\
(\text { SD) }\end{array}$ & $\begin{array}{c}\text { Control Mean } \\
(\text { SD) }\end{array}$ & $\begin{array}{l}\text { Intervention } \\
\text { Mean (SD) }\end{array}$ \\
\hline $\begin{array}{l}\text { Pre-test advertising } \\
\text { literacy }\end{array}$ & 1,4 & $2.90(.47)$ & $2.90(.45)$ & $2.90(.49)$ \\
\hline $\begin{array}{l}\text { Post-test advertising } \\
\text { literacy (raw scores) }\end{array}$ & 1,4 & $3.02(.51)$ & $2.88(.52)$ & $3.11(.48)$ \\
\hline
\end{tabular}

H1: A media literacy intervention can increase advertising literacy when pre-test advertising literacy scores, gender and age are controlled for.

The raw advertising literacy post-test mean score for children in the intervention group $(\overline{\mathrm{x}}=3.1)$ is higher than for children in the control group $(\bar{x}=2.9)$ (see table 1). Model 1 uncovers a statistically significant relationship $(\mathrm{p}<0.001, \mathrm{f}=13.701, \mathrm{df}=4)$ and an Adjusted R2 of $13.6 \%$ indicates that it is a relatively good predictor of advertising literacy.

After controlling for pre-test advertising literacy scores, gender and age, on average a child in the intervention group experienced a greater increase their advertising literacy score $(B=.406,95 \% \mathrm{CI}$ $[0.20,0.61], \mathrm{p}<.001)$, therefore $\mathrm{H} 1$ is accepted. A Hedges $g$ effect size of .406 compares well with earlier studies. These findings further evidence that knowledge domain outcomes can be improved by means of a media literacy intervention, reinforcing the view that children can be empowered to become media literate.

Table 2. Regression models

\begin{tabular}{|c|c|c|c|c|c|c|}
\hline DV & IV & $P$ & $\mathrm{~F}$ & Df & $\begin{array}{l}\text { Adjusted } \\
\mathrm{R}^{2}\end{array}$ & $\begin{array}{l}\text { Beta } \\
\text { (unstandardised) }\end{array}$ \\
\hline Model 1 & & $<0.001$ & 13.701 & 4 & 0.136 & \\
\hline \multirow{4}{*}{\begin{tabular}{l|} 
Advertising \\
Literacy Post- \\
Test Z Score \\
\end{tabular}} & Intervention & $<0.001$ & & & & 0.406 \\
\hline & \begin{tabular}{|l|} 
Advertising \\
Literacy Pre-Test Z \\
Score
\end{tabular} & $<0.001$ & & & & 0.317 \\
\hline & \begin{tabular}{|l|} 
Gender \\
\end{tabular} & 0.092 & & & & -0.173 \\
\hline & Age & 0.526 & & & & 0.065 \\
\hline Model 2 & & $<0.001$ & 11.034 & 5 & 0.134 & \\
\hline \multirow{5}{*}{\begin{tabular}{l|} 
Advertising \\
Literacy Post- \\
Test Z Score \\
\end{tabular}} & Intervention & $<0.001$ & & & & 0.405 \\
\hline & \begin{tabular}{|l|} 
Advertising \\
Literacy Pre-Test Z \\
Score
\end{tabular} & $<0.001$ & & & & 0.366 \\
\hline & \begin{tabular}{|l|} 
Gender \\
\end{tabular} & 0.091 & & & & -0.174 \\
\hline & Age & 0.456 & & & & 0.078 \\
\hline & \begin{tabular}{|l|l|} 
Interaction: & \\
Advertising & \\
Literacy Pre-Test Z \\
Score \\
Intervention \\
\end{tabular} & 0.498 & & & & -0.075 \\
\hline
\end{tabular}

In contrast to the arguments which propose that gender influences susceptibility to marketing messages [50], when all other variables in model 1 were controlled for, gender was not found to have a statistically significant relationship with post-test advertising literacy scores. Similarly, although age has been identified as a pertinent covariate [31, 51; 52], as with gender, this study determines that age was not a predictor of post-test advertising literacy scores when other variables in the model were controlled for. The model depicts that the intervention resulted in a statistically significant increase in the mean advertising literacy score for third- and fourth-class children in the treatment group. Children process an abundance of information via developing cognitive resources. It is imperative that content delivered does not overload these 
resources, otherwise information will be disregarded rather than processed [19]. This study detects no differences in the post-test advertising literacy scores between the classes, indicating that the materials are equally suitable for both age groups. These findings suggest no apparent need to further delineate the teaching materials employed to inform in this regard.

While the addition of the interaction term results in a model (2) that remains statistically significant $(\mathrm{p}<0.001, \mathrm{f}=11.034, \mathrm{df}=5)$, its predictive ability (adjusted $\mathrm{R}^{2}$ ) is marginally lower at $13.4 \%$. The interaction term is not statistically significant $(\mathrm{B}=-$ $.075, \mathrm{p}=0.498$ ). It can be determined that $\mathrm{H} 2$ must be rejected. There is no interaction between the intervention and pre-test advertising literacy scores that helps to predict post-test advertising literacy scores. The intervention is not having a greater effect for those with lower pre-test advertising literacy scores.

\subsection{Process evaluation}

Overall the programme was delivered with relatively high fidelity. During the interviews, time constraints were identified as the key reason for attrition in the number of lessons delivered. The mean lesson preparation time was 16 minutes and the mean lesson delivery time was 49 minutes. The MediaWise resource advises that each lesson should last 40 minutes, therefore the target dosage was 160 minutes. Records of intervention fidelity report that on average 150 minutes of the MediaWise intervention was delivered to classes in the treatment group.

The intervention was well received by both teachers and children. The wide variety of teaching materials was commended by both parties. Children reported that they enjoyed the lessons and wish to see more content. Children particularly enjoyed the worksheets associated with lessons and suggested presenting them in a booklet format. Teachers reported that they found the content fit for purpose, aligned to curriculum and engaging. Furthermore, they reported that the teaching resource was laid out in a user-friendly format. The characteristic challenge of competing needs in modern curriculum was acknowledged. The duration of lessons was deemed lengthy by teachers. Children became very animated when discussing media literacy. In order to reduce the length of time of individual lessons, suggestions made by teachers included reducing the content and reducing the number of worksheets in each lesson.

In the group discussions with children, knowledge of the persuasive intent and the selling intent of marketing messages was apparent. There was also evidence to suggest this knowledge was not enduring. In keeping with the MIP, as the discussion progressed in one focus group, children's affective evaluations of marketing messages discussed superseded their initial cognitive assessments of the message sender's intent. Knowledge of advertisers' motivations and the ability to differentiate between a need and a want did not determine behavioural intention; social motivations to consume supplanted these cognitions. The complex, non-linear, relationship between cognitions, attitudes and intention to behave was apparent. The need for reinforcement of media literacy lessons was demonstrated.

\section{Discussion, Limitations and Future Research}

The aim of the study was to determine the effect of a media literacy intervention on children's advertising literacy. The findings demonstrate that the MediaWise teaching intervention is effective in increasing children's advertising literacy. Those who received the media literacy intervention experienced a greater increase in their advertising literacy scores than those who did not. The findings show that the intervention does not have a greater effect on those with lower pre-test advertising literacy scores, demonstrating that the average benefit observed is universal for children in the treatment group.

It is argued that boys and girls are socialised differently, this study finds no evidence to suggest that advertising literacy is gendered. Though children evidently learn to become consumers as they mature, it should not be assumed that consumer attitudes and skills develop in a linear fashion alongside maturity. Advertising literacy rather than age has a greater impact on consumer behaviour. Children aged between 7 and 12 are well positioned to acquire advertising literacy skills [29]. This analytical stage is key in the development of consumer behaviour as children learn to engage in a variety of decisionmaking strategies, evaluating multiple product attributes simultaneously. This study confirms that it is possible to increase the advertising literacy of children aged 8-11 and it finds no evidence to suggest that it is necessary to delineate this age grouping further. Having the same media literacy curriculum for third and fourth class is a suitable option.

Scope exists to revise and realign the intervention teaching materials so that each lesson time is shortened. A streamlined version of the MediaWise intervention materials will enable teachers to incorporate the intervention into their pedagogy with ease. A smaller set of core activities and worksheets could form an essential booklet, with options for extensions of extra activities or worksheets contained within the overall lesson pack.

Regulation and inoculation are insufficient responses by the adult society who have a duty to 
safeguard and inform children [16] about marketing messages. Media literacy education for children that broadens its focus from the components of media, the communication process and safe online practices, to encompass advertising literacy, will promote the development of cognitive defences and logical heuristics [26]. This will enable children to make informed assessments of messages that are commonly saturated with persuasive appeals. This study documents that a media literacy teaching intervention can improve the advertising literacy of Irish primary school children, corroborating a sizeable body of evidence that argues for the inclusion of media literacy as an essential component of contemporary primary curriculum. These are important findings as research on children's advertising literacy is in its infancy [33]. Data on the effect of a school-based media literacy intervention on advertising literacy in younger children is sparse.

Reinforcement of the classroom learning that has occurred is required. An argument for media literacy lessons forming part of core curriculum is presented. A series of lessons, at regular intervals, will strengthen the learning that has occurred. Children consume a continuous stream of commercial messages. Increased conceptual advertising knowledge will help balance affective assessments of messages that can often override cognitions.

This study measured dispositional advertising literacy as is appropriate given the aim of the intervention is to develop enduring cognitive defences to advertising messages. However, measures of dispositional advertising literacy are limited. This study utilised a measure that addresses key skills of cognitive advertising literacy. While the reliability of the scale employed was acceptable, it could be improved. Further studies could aim to develop a more reliable scale that encompasses a wider measure of dispositional advertising literacy. Moreover, a longitudinal study would prove beneficial in assessing the enduring impact of the intervention on children's levels of advertising literacy and the impact of knowledge on behaviour.

\subsection{Implications for education policy}

Findings from RCTs can help inform education policy [31]. This study shows that a media literacy intervention designed to enable children to evaluate advertising messages can increase children's knowledge of selling intent and persuasive intent. This study also suggests the need for reinforcement of this learning, lending weight to the argument for including media literacy as an integral element of primary curriculum. While the benefits of media literacy are evident, it is unrealistic to expect teachers to afford more time to media literacy education when curriculum is already crowded. Recordings of treatment fidelity evidence the constraints of the curriculum presently and the reality of attempting to adapt existing timetabled hours to include media literacy education. A compelling argument for including media literacy as core curriculum cannot be made without giving the challenge of competing needs due consideration. For change to occur, education policy modification is required to ensure that media literacy education is accommodated. As a starting point within the Irish primary education curriculum, an amendment in the directive from the Department of Education to increase the amount of time afforded to SPHE would enable those teachers who wish to include more media literacy in their teaching to do so. Moreover, if media literacy is designated an essential component of SPHE, a schoolwide collaborative approach could be adopted, similar to that of the Stay Safe programme. To maximise the potential to shape group norms in the school setting, each class could address the same unit (for example Advertising Literacy) simultaneously.

Educators can play a more central role in developing advertising literacy skills in young consumers, enabling increased recognition of stealth marketing messages and informed assessment of marketing appeals, ultimately enabling informed consumer behaviour. These findings are of interest to parties including educators, parents, policy makers and marketers.

\section{References}

[1] Ofcom, Children and parents: Media use and attitudes report, Ofcom, 2018. Available from: https://www.ofcom.org.uk/_data/assets/pdf_file/0024/134 907/Children-and-Parents-Media-Use-and-Attitudes2018.pdf (Last accessed 30 April 2019).

[2] M.R. Nelson, L. Atkinson, M.A. Rademacher, and A. Regina, "How Media and Family Build Children's Persuasion Knowledge", Journal of Current Issues and Research in Advertising, 38(2), 2017, pp. 165-183.

[3] Ofcom, Children and parents: media use and attitudes report, 2015", Ofcom, 2015. Available from: https://www.ofcom.org.uk/_data/assets/pdf_file/0024/785 13/childrens_parents_nov2015.pdf (Last accessed 30 April 2019).

[4] W.J. Potter, Theory of Media Literacy. Sage Publications: United States, 2004.

[5] J. Hill, "Endangered childhoods: how consumerism is impacting child and youth identity", Media, Culture and Society, 33(3), 2011, pp347-362.

[6] M. Tatlow-Golden, L. Tracey and L. Dolphin "Who's feeding the kids online? Digital Food Marketing and Children in Ireland”, Irish Heart Foundation, 2016.

[7] D. Buckingham, S. Banaji, D. Carr, S. Cranmer, and R. Willett, The media literacy of children and young people: a 
review of the research literature, Ofcom, 2015. Available at: http://discovery.ucl.ac.uk/10000145/1/Buckingham medialiteracy.pdf (Last accessed 30 April 2019).

[8] J. Bakan, Childhood under siege: How big business targets your children. New York: Free Press, 2011.

[9] B. O'Neill, "Current policy developments in European media literacy", International Journal of Media and Cultural Politics, 6(2), 2010, pp. 235-241.

[10] European Union, European Media Literacy Week, 2019. Available from: https://ec.europa.eu/digital-singlemarket/en/news/european-media-literacy-week (Last accessed 30 April 2019).

[11] D. Buckingham, R. Willett, S. Banaji and S. Cranmer, "Review of Media Smart 2 Be Adwise: An Evaluation", Centre for the Study of Children Youth and Media, 2007. Available at: https://www.academia.edu/2748245/Media_Smart_Be_Ad wise_2_an_evaluation (Last accessed 30 April 2019).

[12] J. Kenway and E. Bullen, Consuming Children: Education-Entertainment-Advertising. UK: Open University Press, 2003.

[13] B. Šramová, "Media literacy and Marketing Consumerism Focused on Children", Procedia-Social and Behavioral Sciences, 141, 2014, pp. 1025-1030.

[14] M. Easterbrook, M. Wright, H. Dittmar and R. Banergee, "Consumer culture ideals, extrinsic motivations, and well-being in children", European Journal of Social Psychology, 44, 2014, pp. 349-359.

[15] S. Opree, M. Buijzen, and P. Valkenburg, "Lower Life Satisfaction Related to Materialism in Children Frequently Exposed to Advertising", Paediatrics, 130, 2012, pp. 486-491.

[16] United Nations, The UN Convention on the Rights of the Child, 2010. Available from: https://www.childrensrights.ie/sites/default/files/UNCRCE nglish.pdf (last accessed 30 April 2019).

[17] L. Sekarasih, E. Scharrer, C. Olson, G Onut and K. Lanthorn, K., "Effectiveness of a School-Based Media Literacy Curriculum in Encouraging Critical Attitudes about Advertising Content and Forms among Boys and Girls", Journal of Advertising, 0, 2019, pp. 1-16.

[18] P. De Pauw, R. De Wolf, L. Hudders and V. Cauberghe, "From persuasive messages to tactics: Exploring children's knowledge and judgement of new advertising formats", New Media and Society, 20(7), 2018, pp. 2604-2628.

[19] Y. Hwang, J.Y. Yum, and S. Jeong, "What Components Should Be Included in Advertising Literacy Education? Effect of Component Types and the Moderating Role of Age", Journal of Advertising, 47, 2019, pp, 347-361.

[20] M. Friestad, and P. Wright, "The Persuasion Knowledge Model: How People Cope with Persuasion
Attempts", Journal of Consumer Research, 21, 1994, pp. $1-31$.

[21] R. Petty, and J. Cacioppo, "The Elaboration Likelihood Model of Persuasion", Advances in Experimental Social Psychology, 19, 1986, pp. 123-205.

[22] E. Rozendaal, S. Opree, and M. Buijzen, "Development and Validation of a Survey Instrument to Measure Children's Advertising Literacy", Media Psychology, 19(1), 2016, pp. 72-100.

[23] C. Ham, M.R. Nelson, and S. Das, "How to Measure Persuasion Knowledge", International Journal of Advertising, 34(1), 2015, pp. 17-53.

[24] L. Hudders, V. Cauberghe, and K. Panic, "How advertising literacy training affect children's responses to television commercials versus advergames", International Journal of Advertising, 35(6), 2016, pp. 909-931.

[25] E. Rozendaal and S. Opree "The Advertising Literacy of Primary School Aged Children", I. Banks, P. De Pelsmacker, and S. Okazaki, in Advances in advertising research series (Vol. V): Extending the boundaries of advertising, Germany: Springer, 2015, pp. 191-202.

[26] E.W. Austin, B.E. Pinkleton, and R.P. Funabiki, "The Desirability Paradox in the Effects of Media Literacy Training", Communication Research, 34(5), 2007, pp. 483506.

[27] E. Rozendaal, M. Buijzen, and P. Valkenburg, "Comparing Children's and Adults' Cognitive Advertising Competences in the Netherlands", Journal of Children and Media, 4(1), 2010, pp. 77-89.

[28] R. Hobbs, "The State of Media Literacy: A Response to Potter", Journal of Broadcasting \& Electronic Media, 55(3), 2011, pp. 419-430.

[29] S. Livingstone, and E.J. Helsper, "Does Advertising Literacy Mediate the Effects of Advertising on Children? A Critical Examination of Two Linked Research Literatures in Relation to Obesity and Food Choice", Journal of Communication, 56(3), 2006, pp. 560-584.

[30] R. Cartwright, S.J. Opree and A. van Reijmersdal, "Fool's Gold": Linking Materialism to Persuasion Knowledge Activation and Susceptibility to Embedded Advertising', in V. Cauberge, L. Hudders, and M. Eisend, (Eds.), Advances in advertising research series (Vol. IX): Going beyond: Persuading the consumer with new advertising formats, Wiesbaden, Germany: Springer Gabler, 2018, pp. 17-28.

[31] E. Rozendaal, M. Buijzen, and P. Valkenburg, "Children's understanding of advertisers' persuasive tactics", International Journal of Advertising, 30(2), 2011, pp. 329-350.

[32] M.R. Nelson, "Developing Persuasion Knowledge by Teaching Advertising Literacy in Primary School", Journal of Advertising, 45(2), 2016, pp. 169-182.

[33] S. De Jans, D. Van de Sompel, L. Hudders, and V. Cauberghe, "Advertising targeting young children: an 
overview of 10 years of research (2006-2016)", International Journal of Consumer Studies, 40(5), 2017, pp. 601-636.

[34] H. Martens, "Evaluating Media Literacy Education: Concepts, Theories and Future Directions", Journal of Media Literacy Education, 21(1), 2010, pp.1-22.

[35] Buijzen, M., "Reducing Children's Susceptibility to Commercials: Mechanisms of Factual and Evaluative Advertising Interventions", Media Psychology, 9(2), 2007, pp. 411-430.

[36] S. Jeong, H. Cho, and Y. Hwang, "Media Literacy Interventions: A Meta-Analytic Review", Journal of Communication, 62(3), 2012, pp. 454-472.

[37] W.J. Potter, "The State of Media Literacy", Journal of Broadcasting \& Electronic Media, 54(4), 2010, pp. 675696.

[38] B. Walther, R. Hanewinkel, and M. Morgenstern, "Effects of a Brief School-Based Media Literacy Intervention on Digital Media Use in Adolescents: Cluster Randomized Controlled Trial", Cyberphycology, Behaviour and Social Networking, 17(9), 2014, pp. 616623.

[39] DoES, "Wellbeing Policy Statement and Framework for Practice 2018-2023". Department of Education and Skills, 2018. Available at: https://www.education.ie/en/ Publications/Policy-Reports/wellbeing-policy-statement -and-framework-for-practice-2018\% E2\%80\%932023.pdf (Last accessed 30 April 2019).

[40] PDST, Walk Tall, SPHE Curriculum, Professional Development Service for Teachers, 2016. Available from: http://www.pdst.ie/sites/default/files/04\%20Walk\%20Tall $\% 20$ Second\%20Class_0.pdf (Last accessed 30 April 2019).

[41] DoES, "Wellbeing in Primary Schools". Department of Education and Skills, 2015. Available from: https://www.education.ie/en/Publications/EducationReports/Well-Being-in-Primary-Schools-Guidelines-forMental-Health-Promotion.pdf (Last accessed 30 April 2019).

[42] D. Buckingham, "Defining digital literacy - What do young people need to know about digital media?", Nordic Journal of Digital Literacy, 10, 2015, pp. 21-35.

[43] S. McCoy, S. Smyth, and J. Banks, "The Primary Classroom: Insights from the Growing $U p$ in Ireland Study", Dublin: The Economic and Social Research Institute, 2012. Available from: https://www.esri.ie/system/files/media/file-uploads/201510/BKMNEXT205.pdf, (Access date: 23 January 2019).

[44] L.N. Chaplin, P. Hill, and D. R. John, "Poverty and Materialism: A Look at Impoverished Versus Affluent Children", Journal of Public Policy and Marketing, 33(Spring), 2014, pp. 78-92.

[45] D. Buckingham, The Material Child: Growing Up in Consumer Culture, London: Polity Press, 2011.
[46] M.E. Goldberg, G.J. Gorn, L.A. Peracchio, and G. Bamossy, "Understanding materialism among youth", Journal of Consumer Psychology, 13(3), 2003, pp. 278-288.

[47] D. Buckingham, "Media education and the end of the critical consumer", Harvard Educational Review, 73(3), 2003, pp. 309-327.

[48] S. Neeley, "Influences on consumer socialization", Young Consumers, 6(2), 2005, pp. 63-69.

[49] R.W. Belk, "Three scales to measure constructs related to materialism: Reliability, validity, and relationships to measures of happiness", Advances in Consumer Research, 11, 1984, pp. 291 - 297.

[50] M. Dotson, and E. Hyatt, "Major influence factors in children's consumer socialization", Journal of Consumer Marketing, 22(1), 2005, pp. 35-42.

[51] J. Benn, "Consumer education between 'consumership' and citizenship: experiences from studies of young people", International Journal of Consumer Studies, 28(2), 2004, pp. 108 - 116.

[52] G. B. Achenreiner, and D. R. John, "The Meaning of Brand Names to Children: A Developmental Investigation", Journal of Consumer Psychology, 13(3), 2003, pp. 205 - 219.

[53] Professional Development for Service Teachers (PTSD) Walk Tall Programme: Classroom materials to support the SPHE programme, 2016. Available from: http://pdst.ie/walktall, (Access date: 12 February, 2019).

[54] Media Smarts, Teaching Resources, Canada's Centre for Digital and Media Literacy, 2016. Available from: http://mediasmarts.ca/, (Access date: 12 February, 2019).

[55] MediaSmart UK, Teaching Resources, London, 2011. Available from: http://www.mediasmart.org.uk/, (Access date: 12 February, 2019).

[56] Safefood, MediaWise Teaching Resource, Food Safety Authority, Dublin, 2017. Available from: https://www.safefood.eu/Education/Primary(ROI)/MediaWise.aspx, (Access date: 12 February, 2019).

[57] Broadcasting Authority of Ireland, Media Literacy Policy, Dublin: BAI, 2016. Available from: https://www.bai.ie/en/media/sites/2/dlm_uploads/2016/12/ BAI_media_literacy_policy_EN1.pdf, (Access date: 12 February, 2019).

[58] Department of Education and Skills (DoES), Social Personal and Health Education Curriculum, Dublin, 1999.

[59] W. J. Potter, "Guidelines for media literacy interventions in the digital age", Media Research, 20, 2014, pp. 5-31

[60] J. Barker and S. Weller, "Is it fun?" Developing children centred research methods", International Journal of Sociology and Social Policy, 23(1/2), 2003, pp. 33 - 58. 
[61] D. Moher, S. Hopewell, K. Schulz, V. Montori, P. Gøtzsche, P.J. Devereaux, D. Elbourne, M. Egger, D.G. Altman, "CONSORT 2010 Explanation and Elaboration: updated guidelines for reporting parallel group randomised trials”, British Medical Journal, 340, 2010, p. 869.

[62] R. Hobbs, "Measuring the digital and media literacy competencies of children and Teens" in Fran C. Blumberg and Patricia J. Brooks (Eds.), Cognitive Development in Digital Contexts, London: Academic Press, 2017, pp. 253 -274 .

[63] P. Hinton, C. Brownlow, I. McMurray, and B. Cozens, SPSS explained, London: Routledge, 2004.

[64] D.V. Cichetti, "Guidelines, Criteria, and Rules of Thumb for Evaluating Normed and Standardized Assessment Instrument in Psychology", Psychological Assessment, 6(4), 1994, pp. 284-290.

[65] S. Miller, P. Connolly and L.K. Maguire, "A FollowUp Randomised Controlled Trial Evaluation of the Effects of Business in the Community's Time to Read mentoring programme", Centre for Effective Education, Queen's University Belfast, 2011. Available from: http://www.paulconnolly.net/publications/TimeToRead2_f ullreport_2011.pdf, (Access date: 16 January 2019).

[66] Creswell, J., "Qualitative Inquiry \& Research Design: Choosing Among Five Approaches", Thousand Oaks, CA: Sage Publications, 2007. 